more perfect control the various muscles used in the production of voice.

Dr. Scheppegreli-I will ask Dr. Makuen whether he ever heard of the method of a man who traveled over the country a few years ago and made a great deal of money. After insuring his fee, he gave only the direction, "lower your voice." For instance, if the patient used his throat register, he was told to use his chest register. The result really seemed to be to stop the stammering, but I do not know anything as to the permanent regult of the treatment.

Dr. Delavan-In no subject has there been so much quackery and deception as in the cure of stammering. For a man to take up the matter in a scientific, broad-minded and humane way, as Dr. Makuen has done, is quite rare. The mass of literature on the subject does not give a single practical idea. Attention should be given first to the proper management of the breath and the organs of articulation, the proper development of the physical state of the patient, and the careful study of the throat and the removal of any local pathologic defects, adenoid tissue, etc.

Dr. RICHARDS-In the manufacturing community in which I live I find many speech defects among the children. The mothers work in the mills too long previous to the birth of the children and the children themselves are put into the mills very young. These defects are often associated with deficient intelligence, but I have one patient who aside from this trouble is a fairly bright child. The boy can not protrude his tongue and can pronounce no word except his own name, "Elmer," which he can speak intelligibly. At the Massachusetts Gen eral Hospital the trouble was regarded as central in origin There is certainly some hypoglossal paralysis. I would like to know what Dr. Makuen would suggest in a case of this kind.

Dr. CoHEN - In the training of the muscles of the palate the great difficulty is that the soft palate can not occlude the pharynx, and thus it is very rare to see much improvement in speech after operation. For this reason many advisers prefer some artificial appliance. If you ask a patient to choose between an imperfect throat and imperfect speech, he wil usually want his speech restored at almost any cost. It is a very great achievement to get as good results as shown in the first case. The great difficulty in operation is that we have to shorten an already short palate. In 1874 (see Transaction American Medical Association, 1874) I devised an operation for cleft palate, in a girl of 15 . I simply split the edges on each side and spread them out, uniting the two halves. This is the only case where I have seen good speech after operation. I have never performed an operation for cleft palate since, but have invariably advised obturators, and with excellent results. A surgeon once brought me a case on which he had operated for cleft palate, with good results, and he was about to take away the enlarged tonsils. I advised against this procedure, because these tonsils occluded the pharynx and aided speech and their removal would have reproduced the imperfections in speech.

Dr. Woolen -. I would ask whether there is a method of securing adhesion of the two sides of the uvula. In a case I have, strong adhesions of the soft palate were secured, but a bifid uvula remained. Speech has been wonderfully improved, which I think is largely due to the elocutionist to whom I sent the patient after operation, but there are still certain defects in her speech.

Dr. CoHeN-You can get union by putting a drop of nitric acid in the apex of the cleft, and when that spot has united, putting on another drop just below it, and so on.

Dr. Makuen - The average elocutionist does not know what to do with these cases. He has no idea of the proper method of breathing, which is the first thing that has to be taught. I have some assistants in my clinic who understand this subject very well. My elocution teacher in school taught me a method which was entirely wrong. Elocutionists are mainly skilled in teaching the refinements of speech to those who can already speak fairly well. The defective must be shown how to place and use the tongue, lips and palate and the respiratory and buccal muscles, and must be drilled into it. In regard to the various tricks for the cure of stammering, I am sure that I have heard of 500 of them. Few of those who treat stammering will tell you the method used. Dr. Richards mentioned a case of his, a boy who can not protrude his tongue properly. The case I have shown you from Wilmington was one of that kind. The cutting of the anterior fibers of the genio-hyo-glossus muscle relieved him. The operation seems to loosen up the tongue so that the patient can be taught to speak. With reference to the mental condition in these cases, Dr. Daniel G. Brinton says that the genio-hyo-glossus muscle is supposed to have some special connection with the brain, and those who have defective tongue muscles are likely to have a defective brain. I had a case which had been given up as idiotic. I did this operation and the boy is now one of the brightest in his school. It is better to operate under general anesthesia. In stammerers quite a large percentage have bifid uvula.

\section{CHRONIC STENOSIS OF THE LARYNX WITH FIVE ILLUSTRATIVE CASES.}

Presented to the Section on Laryngology and Otology, at the Forty-eighth Annual Meeting of the American Medical Association held at Philadelphia, Pa., June 1-4, 1897.

BY WILLIAM S. JONES, M.D.

CLINICAL PROFESSOR OF LARYNGOLOGY IN THE JEFFERSON MEDICAL COLLEGE HOSPITAL. CAMDEX, N.J.

I reported a case of prolapse of the laryngeal ventricle in the Medical News, Feb. 2, 1895, which presented at intervals marked symptoms of stenosis, the dyspnea at such times being very severe. Since that time, a number of cases of chronic stenosis, due to different causes, have come under my care, and I hope that a short history of some of these may prove both interesting and instructive.

Any cause acting either from within or from without which lessens the lumen of the larynx, will produce the symptoms of stricture, and so long as the cause is active there will be actual constriction. The condition may be acute or chronic, according to the length of time the process has been present and active, but the ultimate symptoms of the two varieties are the same, and the gravity of the symptoms is always dependent upon the severity of the lesion and the extent to which function is interfered with.

The symptomatology is characteristic. Dyspnea is always the most urgent symptom but there may also be present dysphonia, dysphagia, pain, cyanosis from improper aeration of the blood, forced respiration bringing into action the accessory respiratory muscles, anxiety of countenance, engorgement of the venous system and increased breath-sounds in the larynx. In the chronic cases, probably the most frequent initial symptom is dysphonia or hoarseness, which is sooner or later followed by the other characteristic signs.

Case 1.--R. S., aged 35 years, while attending a ball game three years ago, cheered very loudly the success of his favorites. He at once experienced a hoarseness which, however, lasted but a few minutes, and no further attention was given the condition. Two weeks later, hoarseness again developed and gradually increased in severity. After this condition had progressed several weeks, he began to have interference with the breathing which was at times very marked. Four months after the first onset I saw him and found a large mass, of light red color, situated just above the left vocal band and resting upon it. The history of the violent straining of the larynx at the ball game, the immediate hoarseness, probably due to a local congestion, the gradual onset of dysphonia and dyspnea were, in part, characteristic of a prolapsed ventricle, and the appearance of the mass confirmed the diagnosis.

We would state that the diagnosis is by no means easy, and but a very few cases have been reported. The color and smooth surface excluded angioma and papilloma, and the sessile attachment was against myxoma. Its position, shape, color and the absence of surrounding inflammation or infiltration excluded a malignant or tuberculous lesion.

Replacement of the prolapse was performed several times but it returned as soon as pressure was removed, so I resorted to the galvano-cautery to accomplish complete removal. The knife used had a shield to protect the ventricular band. At each visit several applications were made with the knife at a dull-red heat, and after the application of a 20 per cent. solution of 
cocain. The parts healed nicely after complete removal was effected and there are now no appreciable symptoms indicative of any trouble having existed in his larynx. The parts are normal in appearance.

While this case might not be considered one of true stenosis because of the absence of new growth or constricting tissue, it seems to be proper to classify it as such because of the presence of the characteristic symptoms of that condition.

Case 2.-Mr. H. H. A., aged 52 years. The patient was first seen in September, 1896, and the following facts were elicited: The family history and the patient's early record are very good, there being no tuberculous or other constitutional disease present. He has always had good health, the only sickness having been an indefinite venereal trouble thirty years ago from which, however, he quickly recovered. During all these years, he has been engaged in hard labor and exposed to all kinds of weather. He refers the beginning of his present trouble to a bad cold contracted in August, 1895. Dyspnea began in September, 1896, and grew steadily worse until October 12. I first saw him early in September. At that time he had slight hoarseness, and examination showed a small mass, the color of the normal larynx, beneath the left vocal cord. He was placed upon specific treatment tentatively. He grew steadily worse. Marked dyspnea developed and almost complete aphonia, and all the usual symptoms of advanced stenosis. October 12, examination of the larynx showed marked thickening of the left ventricular band and bulging of the left cord, caused apparently by a swelling back of it. The swelling was so great that breathing was almost impossible. The right side was normal. Tracheotomy was performed at once.

The operation was done under eucain anesthesia and the third, fourth and fifth rings cut. Three days after the operation he contracted an acute bronchitis, which however subsided in a few weeks and he left the hospital at the end of a month. One month later he began to breathe through the mouth, also to speak, and in two months his voice was perfectly normal and his breathing free. He still wears his tube but keeps it plugged all the time.

The cause of the stenosis in this case was obscure. There was no ulceration and no pulmonary involvement, which, together with his recovery without medication, exclude tubercular laryngitis, and syphilis was excluded by the week's trial treatment. The probable cause was a localized chondritis which subsided after the irritation by breathing, coughing and talking was removed. This also seems probable from the fact that at present the larynx is normal in every respect.

Case 3.-W. W., aged 42 years. His family history is negative excepting that one brother died at the age of 21 of acute miliary tuberculosis. His personal history reveals that he had three chancroids (so-called) in 1872, which were about three months in healing. He, however, never saw any secondary symptoms of syphilis, but as he is colored they may have been present and unnoticed. In 1891, he was kicked by a horse in the right side of the face. The malar and superior maxillary bones were fractured, and a portion of the latter was removed by the surgeon in attendance. About three weeks after this injury, according to his statement, a sore throat developed and has continued more or less ever since. In 1893, an abscess formed in the left side of his neck and was opened. This probably came from broken down cervical glands, as a number of them in that region are still enlarged. About the middle of July, 1896, he began to have dyspnea and difficulty in swallowing solid foods, with occasional attacks of vomiting. These symptoms gradually increased until the middle of October when I saw him. For a week previous to this time dyspnea was very marked, and he had some pain in both tonsillar regions.

October 25, I performed tracheotomy under eucain anesthesia. He made a slow recovery from the operation, having much inflammatory reaction about the wound. He was given 1.3 grams of potassium iodid three times a day immediately after the operation, and this has been kept up until the present time. The condition of the larynx remained unchanged for four months in spite of the administration of the iodid, but then the obstruction began to disappear and at present (May 1) his larynx is almost normal and his voice is completely restored.
This case was undoubtedly one of syphilis of the larynx and the favorable result is due in part to the early operation, removing a fertile source of irritation by turning the current of air aside and preventing coughing, and in part to the steady use of the iodid before the new tissue was thoroughly organized, as were organization complete, a contracting stricture would undoubtedly have resulted.

Case 4.-P. B., aged 53 years. His family history was very good, also his personal history, he having had no sickness except the grip three years ago. His present trouble began about Sept. 1, 1896, with slight dyspnea which gradually increased, but he continued his work as a laborer until October 8. As his difficulty of breathing became more severe he developed a cough, but had no pain or difficulty in swallowing. He lost flesh rapidly during this time. He first came to the clinic October 27 and an examination then revealed a growth involving the right side of the larynx and pushing the vocal band to and beyond the middle line. The diagnosis of carcinoma was made and immediate tracheotomy advised, with a probable resort to laryngectomy later. The first operation was done the next day under eucain anesthesia. He recovered quickly from the operation but continued to lose flesh. The laryngeal swelling subsided somewhat after the operation but the growth increased steadily in size. He refused laryngectomy and went to his home in a neighboring city, where he is still living, but in the last stages of malignant disease.

Case 5.-W. C., aged 30 years, a laborer. Nov. 12, 1896, in a fit of despondency, he attempted suicide by making a large cut in his throat with a penknife. He penetrated the trachea about the second or third ring, twisted the blade about and cut up and down for an inch or more. He was taken to a hospital, where the wound was dressed but not closed, nor was there a tube inserted. One week later, it was noticed that he could not breathe through the mouth when the wound was closed by pressure. It was deemed necessary to introduce a tracheotomy tube, an anesthetic was given him, a tube inserted and the wound stitched around it. Two weeks later, the surgeon attempted to pass a tube through the trachea and larynx from the tracheal wound but did not succeed, the part being shut off. He was then sent home to allow the wound to heal before further operative interference was instituted. Nothing further, however, was done and he was sent to me. The condition of his larynx at present is as follows: The right side is drawn slightly down ward, the right vocal band is in the median line and immovable, being fixed by scar tissue beneath it; the left side is normal in situation, the cord is freely movable and can be brought to the middle line perfectly. The entire left side is free for three-fourths of an inch below the vocal band, while the right side has a band of cicatricial tissue just below the cord, which extends downward for three-fourths of an inch and then spreads out to the other sides, completely occluding the trachea. The surface of this membrane is smooth and the lowest point is near the center. From below, the trachea is entirely closed above the tube and the cicatrix is about onehalf an inch in thickness.

I have made no attempt at opening the trachea but think it can be done comparatively easily from above. The method of procedure will be to make an incision through the scar tissue with a long knife and enlarge this opening by graduated bougies until the entire lumen is established, and then keep it open by a tube attached to the one now in the tracheal wound until the surrounding parts are healed. He might even always wear an intratracheal tube, as it is too low to interfere with his voice, yet will insure an open passage for air.

201 Broadway.

\section{SOGIETY PROCEEDINGS.}

\section{New York Academy of Medicine-Section in Orthopedic Surgery. \\ Meeting of December 1\%, 1897.}

Dr. A. M. Phelps read a paper entitled:

A CONSIDERATION OF SOME OF THE PATHOLOGIC AND MECHANICAL PROBLEMS OF HIP DISEASE.

He presented the view that nature attempted to repair the lesion producing hip disease by inflammatory action which was a normal process of repair until the inoculation of germ life which marked the beginning of disease in the area of inflammation. The absence of inoculation gave rise to the ephem. 